\title{
USING AGENTS' ATTITUDES AND ASSESSMENTS IN AUTOMATED FUZZY BIDDING STRATEGY
}

\author{
Madhu Lata Goyal, Jun Ma \\ Faculty of Engineering and Information Technology, University of Technology, Sydney, Australia \\ madhu@it.uts.edu.au,junm@it.uts.edu.au
}

Keywords: $\quad$ Fuzzy Sets, Agent's Attitudes, Bidding Strategy .

Abstract: $\quad$ To be successful in multi-attribute auction, agents must be capable of adapting to continuous changing bidding price. This paper presents a novel fuzzy attitude based bidding strategy (FA-Bid), which employs dual assessment technique i.e. assessment of multiple attributes of the goods as well as assessment of agents attitude (eagerness) to procure an item in automated auction. The assessment of attributes adapts the fuzzy sets technique to handle uncertainty of the bidding process as well use heuristic rules to determine attitude of bidding agents in simulated auctions to procure goods. The overall assessment is used to determine a price range based on current bid, which finally selects the best one as the new bid.

\section{INTRODUCTION}

The emergence of electronic market places has dramatically increased the opportunities for the online auctions (e.g. eBay, Amazon etc.). Intelligent agent technology [Anthony and N.R.Jennings, 2002, Byde et al., 2002, Greenwald and Stone, 2001, He et al., 2003] provides a powerful mechanism to address complex problems of dynamic pricing in automated auctions. The agents can use different auction mechanisms (e.g. English, Dutch, Vickery etc.) for procurement of goods or reaching agreement between agents. The agent makes decisions on behalf of consumer and endeavours to guarantee the delivery of item according to the buyers preferences. In these auctions buyers are faced with difficult task of deciding amount to bid in order to get the desired item matching their preferences. For this reason, the formalisation of bidding mechanism has received a great deal of attention from the agent community for the past decade. These software agents should be smart enough to bargain a favourable deal for the user. In order to be called an intelligent agent the software must satisfy several criteria like autonomy, temporal continuity, communication and cooperation. To this end, a number of researchers [P.Anthony and N.R.Jennings, 2003, Kowalcyzk and Bui, 2000, Luo et al., 2003, Ma and Le- ung, 2007,P.Stone et al., 2001] have reported different frameworks that help an autonomous agent to tackle the problem of bidding in auctions. Currently, no single implementation satisfies all the criteria, but there are several promising results for bargaining intelligent agents.

In this paper, a fuzzy bidding strategy (FA-Bid) is designed in an automated auction based on the dual assessment of multiple attributes of items as well as agents attitude on bidding item. To quantify attitudes and to deal with uncertainty of attribute assessment fuzzy sets technique is applied in the presented strategy. The basic procedure of the strategy is shown in Figure 1. The remainder of the paper is organized as below. First, the detail of the presented strategy is illustrated. Then, a simple experiment is conducted. Related work and conclusion are discussed finally.

\section{A FUZZY BIDDING STRATEGY (FA-BID)}

In an automated auction, an agent's bidding activity is influenced mainly by two aspects, namely, 1) the attributes of goods and 2) the agent's attitude. Any agent prefers to make a bid for a quality goods. Rais- 


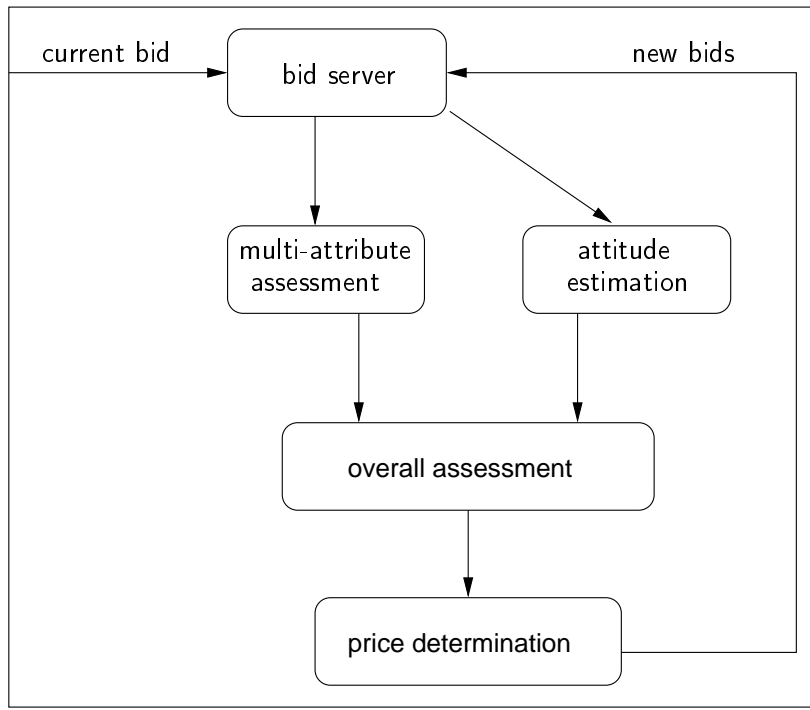

Figure 1: A Fuzzy Bidding Strategy (FA-Bid) model.

ing bids will dampen the established attitude of an agent on the goods. All these facts require an intelligent agent system, plays the role of an agent's representation, to adopt an appropriate bidding strategy. Considering the existence of uncertainty in a real auction situation, this paper focuses on how to make bid by using the agent's personal perspective.

To make a bid for a unit of goods, the agent should balance between his/her assessment on the goods and his/her attitude (aspiration) to win an auction. Generally speaking, an agent has stronger eagerness to make bid for a quality goods rather than a lower one. The eagerness is mainly based on the assessment on the goods. Moreover, an agent's attitude is also influenced by the bids because price is the unique factor through which agents and an auctioneer negotiate till make a deal. To win an auction, an agent must balance among the price (bid), assessment on the goods and attitude to win a bid.

Roughly speaking, the bidding procedure runs as follows:

- Firstly, evaluation on each related attributes is determined.

- Then these evaluations are aggregated to form an overall assessment on the goods.

- Next, the attitude of the agent is determined.

- Overall assessment is conducted.

- Finally, a new bid is determined.

Since in real situation uncertainty exists ubiquitously in expressing assessments, eagerness as well as their relationships with price, this paper uses fuzzy- set-based method to process uncertainty in assessment and eagerness. First of all, this paper uses a satisfactory degree measure as the common universe of assessment, i.e., an assessment is treated as a fuzzy set on the satisfactory degree. Secondly, an eagerness is expressed as a fuzzy set on the set of assessments, i.e., the assessment set is the universe of eagerness.

In the following sections, details of the strategy is illustrated.

\subsection{Attribute Evaluation}

Attribute evaluation includes two kinds of process. The first one is individual attribute assessment, and the second one is assessment aggregation. To implement attribute evaluation, three issues are concerned, i.e., attribute weights (relative importance) adjustment, assessment expression, and assessment aggregation.

\subsubsection{Weights adjustment}

Weight adjustment implements dynamically change relative importance of multiple criteria. In a real situation an agent's personal preference on the attributes seldom has quickly fluctuation, i.e., the weights for criteria is relatively stable in a long run. The adjustment of weights resulted from the price should be limited to a rational range. Moreover, the adjustment shouldn't change the relative significance among criteria other than the price because raising price alters the relative significance of it to other criteria. In the following, the agent's preference is treated as an initial weight vector which is the basis of the adjustment. To construe an initial weight vector, the Analytic Hierarchy Process (AHP) method [Saaty, 1980] is applied because it is proved validate in practice although it may induce inner inconsistency. Suppose the obtained initial weight vector is $W^{(0)}$.

Suppose the current bid $p_{c}$ belongs to $\left[p_{l}, p_{u}\right] \subseteq \mathbb{R}$ where $p_{l}$ and $p_{u}$ are the lower and upper boundaries of possible bids respectively which are determined by the auction. Let $C=\left\{c_{0}, c_{1}, \ldots, c_{K}\right\}$ be the set of $K+1$ attributes and $W=\left\{w_{0}, w_{1}, \ldots, w_{K}\right\}$ is the set of weights for attributes in $C$.

Because except the price agent's assessments on other criteria do not change, the adjustment of weight for price should be determined first. Suppose $[-\delta, \delta]$ is the adjustable range of the weight for price and the current net increasing of weight for price is $\Delta w_{0}$, then 
the current weight vector is determined by

$$
\begin{aligned}
& w_{0}^{\prime}=w_{0}+\Delta w_{0} \\
& w_{k}^{\prime}=w_{k} \cdot \frac{1-w_{0}^{\prime}}{1-w_{0}}, \quad k=1,2, \ldots, K .
\end{aligned}
$$

where $w_{k}(k=0,1, \ldots, K)$ is the component of $W^{(0)}$. Obviously,

$$
\sum_{k=0}^{K} w_{k}=1,
$$

and the relative significance of the criteria except for the price will not change after this adjustment.

\subsubsection{Assessment Expression}

Since uncertain expressions are often used in a real situation, this paper uses linguistic terms to express assessments. These linguistic terms are illustrated by fuzzy set. Moreover, the universe of these fuzzy set are unified to real interval $[0,1]$ which means the satisfactory degree of the agent to a particular attribute. Therefore, all fuzzy sets have same universe which is convenient for aggregating assessments.

Suppose $g_{k}(k=0,1, \ldots, K)$ is the satisfactory degree measure for attribute $c_{k}$. Then an agent's opinion on the goods in terms of attribute $c_{k}$ is denoted by $g_{k}(u)$ where $u\left(\in U_{k}\right)$ is the real attribute value of attribute $c_{k}$ and $U_{k}$ is the real universe for attribute $c_{k}$. For instance, departing time is an attribute for a flight ticket. The possible departing time in a day is from $0: 00$ to $23: 59$. For any time slot $u$, a client may present a satisfactory degree such as departing at $7: 30$ is with satisfactory degree 0.9 and departing at $3: 00$ is with 0.3 .

In the following, let $A=\left\{a_{1}, \ldots, a_{n}\right\}$ be the set of used assessment terms which are fuzzy sets on satisfactory degree $[0,1]$. Then a numeric satisfactory degree is transformed to a linguistic term. Continue the above example, suppose the assessment set is as shown in Figure 2. Notice that $a_{7}$ is with the biggest the membership degree for 0.9 , the assessment for departing at $7: 30$ is $a_{6}$ by the maximum membership degree principle. Similarly, the assessment for 0.3 is $a_{2}$.

\subsubsection{Assessments Aggregation}

An aggregated assessment is the agent's overall opinion/preference on the goods in terms of multiple attributes. Take booking a flight ticket for example, an assessment is made on a ticket usually based on the airlines, flight departure and arrival time, flight type, aircraft types, seat positions, as well as price. The change of an attribute's value may leads to the

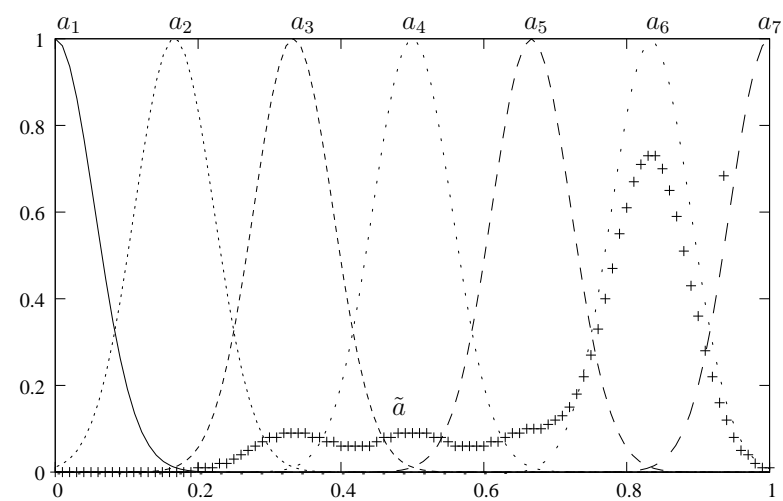

Figure 2: Obtain overall assessment.

alternation of an assessment. Instinct natures of different attributes increase the difficulty and uncertainty for obtaining an overall assessment. Notice that an agent's preference on an individual attribute can be expressed through the agent's satisfactory degree on that attribute. This paper uses an satisfactory degree measure as the common universe of assessment.

Based on assessment on each individual attribute, an overall assessment can be obtained as follows. Suppose the individual assessments of all attributes are $v_{0}, v_{1}, \ldots, v_{K}$ and the weights of them are $w_{0}, w_{1}$, $\ldots, w_{k}$ respectively. Then an overall assessment is obtained by

$$
a=\operatorname{Agg}\left\{\left(v_{0}, w_{0}\right),\left(v_{1}, w_{1}\right), \ldots,\left(v_{K}, w_{K}\right)\right\}
$$

where Agg is a selected aggregation method, $v_{k} \in A$ $(k=0,1, \ldots, K)$ is the linguistic assessment on attribute $c_{k}$.

To get an overall assessment in terms of a set of criteria, an aggregation method Agg is applied. Some existing methods can be used here, such as OWA operator [Yager, 1993, Yager, 2004], 2-tuple linguistic aggregation [Delgado et al., 1999, Delgado et al., 2001, Herrera et al., 2001], and Weighted-sum. For convenience, we use the weighted-sum-based method to obtain an overall assessment as follows.

First, we construct a fuzzy set $\tilde{a}$ on $[0,1]$ through

$$
\tilde{a}(u)=\sum_{k=0}^{K} w_{k} \cdot v_{k}(u), \quad u \in[0,1],
$$

where $v_{k}(u)$ is the membership degree of $u$ in $v_{k}$.

Next, we calculate the distance between $\tilde{a}$ and $a_{i} \in$ $A$ by

$$
d\left(\tilde{a}, a_{i}\right)=\int_{0}^{1}\left|\tilde{a}-a_{i}\right| \mathrm{d} \lambda .
$$

Finally, we select the nearest term(s) $a$ to $\tilde{a}$ as the overall assessment. 
For example, $A$ has seven terms, namely, $a_{1}, a_{2}$, $\cdots, a_{7}$ as shown in Figure 2. Suppose $\tilde{a}$ is the obtained fuzzy set. By comparing the distances between $\tilde{a}$ and each element in $A$, we know $a_{6}$ is the nearest item to $\tilde{a}$. Hence, $a_{6}$ will be taken as the overall assessment.

\subsection{Attitude Estimation}

Customer's attitude is his or her willingness to bid for a unit of goods, which is related to but not the same as the overall assessment on the given goods. After conducting new assessment on the goods according to current price $p_{c}$, estimation of agent's attitude is implemented. In order to do so, the relationship between attitude and assessments is required. In general, the better the assessment on the given goods is, the stronger the attitude of bidding for that goods will be. However, this is by no means the unique relationship between attitude and assessment. For instance, other agents' competitive bidding sometimes can also cause strong willingness. In this paper, we mainly focus on the factor of assessment and extract this relationship from the agent's transaction records.

Suppose $E=\left\{e_{1}, \ldots, e_{m}\right\}$ is the set of attitude expressions, $A=\left\{a_{1}, \ldots, a_{n}\right\}$ is the set of assessments, and $T=\left\{t_{1}, \ldots, t_{L}\right\}$ is the agent's transaction records such that $t_{i}=1$ if the client won the transaction $t_{i}$, otherwise $t_{i}=0$. Because in each transaction, the agent's assessment and attitude occur simultaneously, a set of formal rule, denoted by $R$, thus can be extracted from $T$ such that any $r \in R$ is of form

$$
r: \quad\left(a_{i} \Rightarrow e_{j}, \alpha_{i j}\right),
$$

where $a_{i} \in A, e_{j} \in E$, and $\alpha_{i j}$ is the reliability degree obtained by

$$
\alpha_{i j}=\frac{\mid\left\{t \in T \mid a_{i}, e_{j} \text { occur in } t \text { and } t=1\right\} \mid}{\mid\left\{t \in T \mid a_{i} \text { occurs in } t \text { and } t=1\right\} \mid} .
$$

Such rule depicts the approximate degree of agent's attitude $e_{j}$ to which the agent can win the bid under the assumption that the overall assessment is $a_{i}$. Furthermore, these rules can be treated as a set of fuzzy sets on $A$ such that the membership degree in a fuzzy set $f_{j}$ corresponding to eagerness $e_{j}$ is $\alpha_{i j}$. Obviously, $f_{j}$ is an integration of rules $\left(a_{i} \Rightarrow e_{j}, \alpha_{i j}\right)$ $(i=1, \ldots, n)$, which is able to be treated as an alias of $e_{j}$. Hence, the fuzzy set $f_{j}$ is also called attitude in the following without other specification.

Based on the rules in $R$, an agent can estimate the possible attitude of the agent when it learns the current overall assessment. set of fuzzy sets is obtained through the following way: suppose the overall assessment is $a_{c}$, then the attitude at the moment is determined by the maximum membership degree prin- ciple

$$
e_{c} \in E\left(a_{c}\right)=\left\{e_{j} \in E \mid f_{j}\left(a_{c}\right) \geqslant f_{i}\left(a_{c}\right) \text { if } i \neq j\right\} .
$$

Notice that such determined $e_{c}$ may not necessarily be unique. In the following, we call $E\left(a_{c}\right)$ the candidate attitude set under $a_{c}$.

Once the current attitude of the agent is determine, requirements for search new bids can then be determined. The main requirements include identifying required overall assessment and finding the candidate prices.

\subsection{Overall assessment}

Prerequisite of overall assessment is the basic requirement on the goods such that the agent has the highest possibility to win a bid under the current attitude.

To find the prerequisite of overall assessment, an order is firstly defined in $E$ according to the strength of attitude. Without loss of generality, suppose $e_{i}<e_{j}$ if $i<j$. Therefore, it is possible to select the strongest element from $E\left(a_{c}\right)$. Then the strongest element in $E\left(a_{c}\right)$ is chosen as the first candidate attitude to determine the prerequisite of overall assessment. From the agent's transaction records, a set of rules $\bar{R}$ is determined such that any $\bar{r} \in \bar{R}$ is of form

$$
\bar{r}: \quad\left(e_{j} \Rightarrow a_{i}, \bar{\alpha}_{i j}\right),
$$

where $e_{j} \in E, a_{i} \in A$, and $\bar{\alpha}_{i j}$ is the reliability degree obtained by

$$
\bar{\alpha}_{i j}=\frac{\mid\left\{t \in T \mid a_{i}, e_{j} \text { occur in } t \text { and } t=1\right\} \mid}{\mid\left\{t \in T \mid e_{j} \text { occurs in } t \text { and } t=1\right\} \mid} .
$$

Based on the maximum membership degree principle, a set of candidate assessment is determined such that

$$
A\left(e_{c}\right)=\left\{a_{i} \in A \mid \bar{f}_{i}\left(e_{c}\right) \geqslant \bar{f}_{j}\left(e_{c}\right) \text { if } i \neq j\right\},
$$

where $\bar{f}_{i}$ is the counterpart to $f_{i}$. Each element $a$ in $A\left(e_{c}\right)$ is called a candidate assessment under eagerness $e_{c}$.

\subsection{Agent price determination}

An agent's assessment demonstrates some expection on the quality of the goods. As other criteria except the price are seldom changeable in an auction, this is regarded in terms of price.

Suppose $U_{0}=\left[p_{l}, p_{u}\right]$ is the real range of price. A price range $U(a)$ corresponding to a candidate assessment $a$ is a subset of $U_{0}$ such that for any $u \in U(a)$, the assessment based on $u$ and $W$ is $a$. Notice that an assessment is a fuzzy set on the satisfactory degree $[0,1]$ 
which is the bridge between assessment and price, a price range is determined by the following steps.

Step 1: We divide the satisfactory degree $[0,1]$ into $n$ subsets $D_{1}, D_{2}, \ldots, D_{n}$ such that

$$
a_{i}(d) \geqslant a_{j}(d)
$$

for any $d \in D_{i}$ and $j \neq i$, i.e., element in $D_{i}$ with biggest membership degree in $a_{i}$.

Step 2: For $D_{a}$ corresponding to a candidate assessment $a$, we select price in $U_{0}$ such that $g_{a}(u) \in D_{a}$. $U_{a}$ is called a candidate bid set. Concerning that the satisfactory degree is continuously change with the price, we assume that $U_{a}$ is an interval in $U_{0}$. Hence, let $p_{l a}$ and $p_{u a}$ be the left and right boundary of $U_{a}$.

Thus, a candidate price range for assessment $a$ is determined.

Suppose for any element in $A\left(e_{c}\right)$, we have obtained a corresponding candidate price range. Because new bid should higher than the present price $p_{c}$, a candidate price set for $A\left(e_{c}\right)$ is determined by

$$
\begin{aligned}
U_{A}= & \left\{p_{l i} \mid p_{l i}>p_{c}, a_{i} \in A\left(e_{c}\right)\right\} \\
& \cup\left\{p_{u i} \mid p_{u i}>p_{c}, a_{i} \in A\left(e_{c}\right)\right\} .
\end{aligned}
$$

As it can be seen that the candidate price range may not exist under some assessments, in these case, a weaker attitude is selected to repeat the candidate price determination process until a range is found or the attitude is weaker than an acceptable level.

Suppose $U_{A}$ is a found price range, there must be a smallest element $b$ in it. Then $b$ is selected as the new bid.

\section{EXPERIMENT EVALUATION}

In this section, an experiment implements the fuzzy bidding strategy in a scenario in which an agent intends to book flight tickets. Six factors (as shown in Table 1) are concerned in this situation, i.e. ticket price $\left(c_{0}\right)$, depart time $\left(c_{1}\right)$, arrival time $\left(c_{2}\right)$, number of stops $\left(c_{3}\right)$, seat positions $\left(c_{4}\right)$, and travel season $\left(c_{5}\right)$. The flight ticket bid for is a return ticket to destination $D$ with the following properties:

- price: $\$ 800$ - $\$ 2000$;

- depart time: 18:00 PM, Wednesday;

- return arrival time: 10:00 AM, Friday;

- number of stops: 1 ;

- seat position: window;

- travel season: April (off-peak season).

Suppose the identified perspective of an agent is summarized as below:
Table 1: Concerned attributes of a flight ticket.

\begin{tabular}{lclr}
\hline Attributes & Symb. & Values range & Weights \\
\hline price & $c_{0}$ & $\$[800-2000]$ & 0.4 \\
depart time & $c_{1}$ & Sun. 0:00 - Sat. 24:00 & 0.1 \\
arrival time & $c_{2}$ & Sun. 0:00 - Sat. 24:00 & 0.1 \\
stops & $c_{3}$ & 0, 1, 2, 3 & 0.1 \\
seat position & $c_{4}$ & window, aisle, middle & 0.1 \\
flight season & $c_{5}$ & Jan. 01 - Dec. 31 & 0.2 \\
\hline
\end{tabular}

- The agent prefers to a cheaper ticket and agrees to that the cheaper the better.

- The agent prefers to travel at the weekend rather than at working day.

- The agent prefers to no stop travel.

- The agent prefers to aisle seat then window seat.

- The agent prefers to travel during off-peak season rather than peak season.

- The agent thinks the flight price is the most important factor, secondly the travel season, and other factors are of same importance.

Based on the agent's perspective, the agent evaluates the ticket using seven terms (shown in Figure 3), i.e., very bad $\left(a_{1}\right)$, bad $\left(a_{2}\right)$, slightly bad $\left(a_{3}\right)$, acceptable $\left(a_{4}\right)$, fairly good $\left(a_{5}\right)$, good $\left(a_{6}\right)$, and very good $\left(a_{7}\right)$. The seven terms are expressed by fuzzy sets on the satisfactory degree $[0,1]$ as below (see Figure 3 ):

$$
f_{a_{i}}=e^{-162\left(x-(i-1) \frac{1}{6}\right)^{2}}, \quad i=1, \ldots, 7 .
$$

The assessment on each individual factor is

\begin{tabular}{cl}
\hline Attribute & Assessment \\
\hline$c_{0}$ & (no assessment) \\
$c_{1}$ & good $\left(a_{6}\right)$ \\
$c_{2}$ & fairly good $\left(a_{5}\right)$ \\
$c_{3}$ & slightly bad $\left(a_{3}\right)$ \\
$c_{4}$ & acceptable $\left(a_{4}\right)$ \\
$c_{5}$ & good $\left(a_{6}\right)$ \\
\hline
\end{tabular}

As the ticket price is the changeable factor, the assessment on it is determined dynamically. For convenience, suppose the agent's satisfactory degree measure on price is expressed by a linear function as below:

$$
g_{0}(p)=\frac{2000-p}{1200}
$$

Now assume the current price $\left(p_{c}\right)$ is $\$ 900$, the agent is required to determine a new bid in this situation.

First, the satisfactory degree of the current price is calculated by Eq. (15), which is 0.91. Because $f_{a_{7}}(0.91)=0.35$ and $f_{a_{6}}(0.92)=0.82$, the assessment for $p_{c}$ is $a_{6}$ (good). 


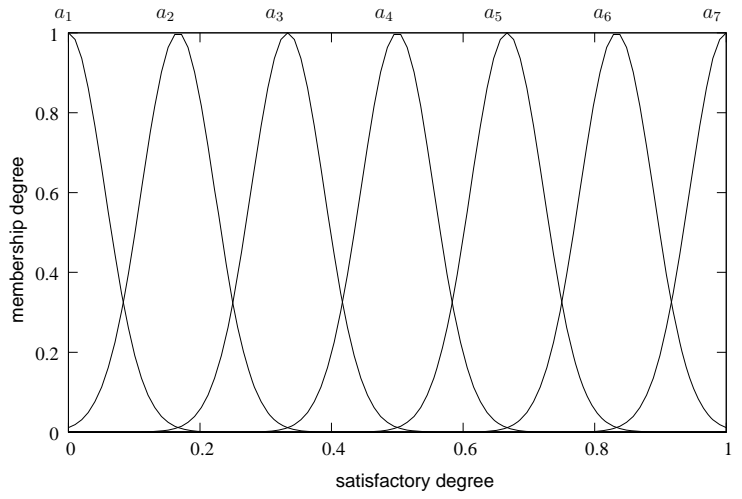

Figure 3: Assessment terms.

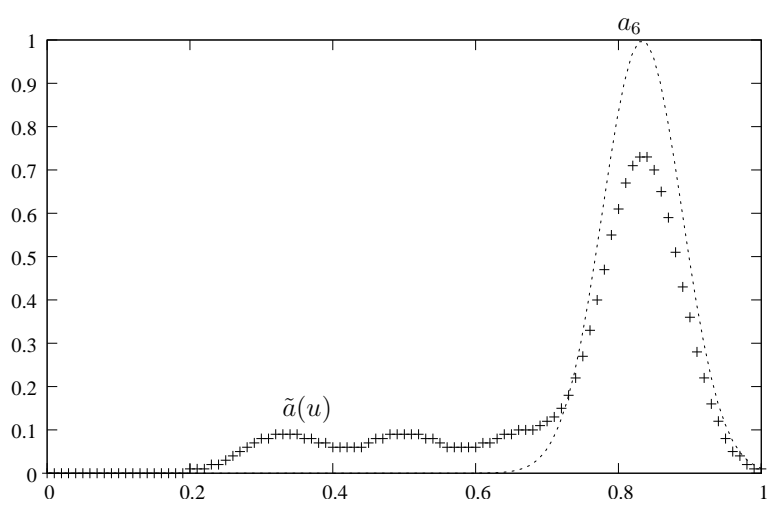

Figure 4: Overall assessment.

Next, since the price changes will affect the weights of all factors, a new overall assessment of the ticket is calculated. Suppose the increase of price weight is 0.05 , i.e., the current weight of price is $w_{0}^{\prime}=0.45$. Then the weights of other factors are calculated by Eq. (2), and they are

$$
\begin{aligned}
w_{1}^{\prime}=w_{2}^{\prime}=w_{3}^{\prime}=w_{4}^{\prime} & =0.09 \\
w_{5}^{\prime} & =0.19 .
\end{aligned}
$$

Therefore, a fuzzy set $\tilde{a}(u)$ is obtained $(\tilde{a}(u)$ in Figure 4). Then by Eq. (6), the most nearest assessment to $\tilde{a}$ is $a_{6}$. So the new overall assessment for the ticket is $a_{6}$.

Then the agent needs to estimate the agent's attitude according to this assessment. Suppose the agent uses five terms to distinguish the attitude, i.e., none $\left(e_{1}\right)$, slightly $\left(e_{2}\right)$, medium $\left(e_{3}\right)$, strong $\left(e_{4}\right)$, and very strong $\left(e_{5}\right)$. In order to estimate the agent's attitude, a set of rules of form Eq. (7) are extracted from a historical auction records, which are illustrated in Table 2 and Figure 5.

By Figure 5, the agent's attitudes at this moment are $e_{2}$ and $e_{3}$ because they have the highest reliability.
Table 2: Rule set for attitude estimation.

\begin{tabular}{c|ccccc}
\hline & \multicolumn{5}{|c}{ attitude } \\
\cline { 2 - 6 } ass. & $e_{1}$ & $e_{2}$ & $e_{3}$ & $e_{4}$ & $e_{5}$ \\
\hline$a_{1}$ & 0.17 & 0.23 & 0.20 & 0.27 & 0.13 \\
$a_{2}$ & 0.10 & 0.28 & 0.22 & 0.26 & 0.13 \\
$a_{3}$ & 0.10 & 0.26 & 0.18 & 0.32 & 0.13 \\
$a_{4}$ & 0.17 & 0.26 & 0.23 & 0.23 & 0.12 \\
$a_{5}$ & 0.12 & 0.25 & 0.27 & 0.21 & 0.16 \\
$a_{6}$ & 0.12 & 0.26 & 0.26 & 0.23 & 0.13 \\
$a_{7}$ & 0.12 & 0.24 & 0.31 & 0.24 & 0.10 \\
\hline
\end{tabular}

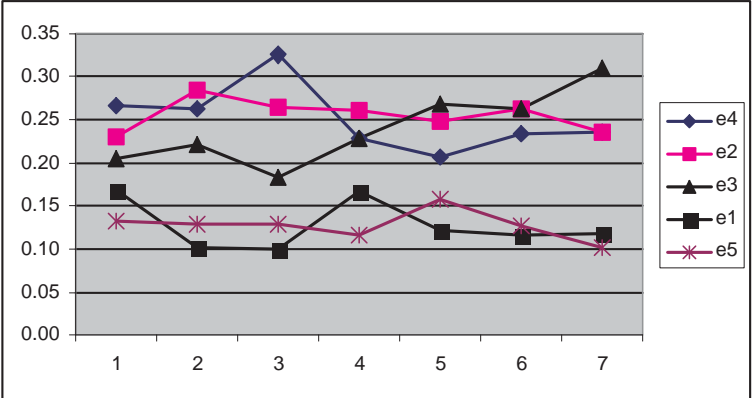

Figure 5: Illustration for rule set $R$.

Because $e_{3}$ is stronger than $e_{2}$, the agent first searches possible bids under the attitude $e_{3}$. Based on $e_{3}$, the agent discovers that $a_{6}$ is the most preferred assessment on the ticket through rules in Table 3. Hence, it will determine a candidate price range based on the assessment $a_{6}$.

Based on Figure 3, the agent can divide the satisfactory degree interval $[0,1]$ into seven sub-intervals. In this figure, the interval corresponding to assessment $a_{6}$ is $\$[900-1100]$ and the current price $p_{c}$ belongs to this interval. Hence, a new bid can be selected from the interval. According to the FABstrategy, the smallest one greater than the $p_{c}(900)$ will be selected. For instance, if the least increase is $\$ 50$, then the new bid $b$ is $\$ 950$.

Table 3: Rule set for prerequisite of assessment identification.

\begin{tabular}{c|ccccccc}
\hline & \multicolumn{7}{|c}{ assessment } \\
\cline { 2 - 8 } att. & $a_{1}$ & $a_{2}$ & $a_{3}$ & $a_{4}$ & $a_{5}$ & $a_{6}$ & $a_{7}$ \\
\hline$e_{1}$ & 0.11 & 0.16 & 0.12 & 0.24 & 0.16 & 0.16 & 0.06 \\
$e_{2}$ & 0.07 & 0.21 & 0.15 & 0.18 & 0.16 & 0.17 & 0.06 \\
$e_{3}$ & 0.07 & 0.18 & 0.11 & 0.17 & 0.18 & 0.19 & 0.09 \\
$e_{4}$ & 0.09 & 0.20 & 0.19 & 0.16 & 0.13 & 0.16 & 0.06 \\
$e_{5}$ & 0.08 & 0.19 & 0.15 & 0.16 & 0.20 & 0.17 & 0.05 \\
\hline
\end{tabular}




\section{CONCLUSIONS}

In this paper, a novel attitude-based agent's bidding strategy (FA-Bid) is discussed. It was noticed that agents, which adopt attitudes, behave more flexibly and efficiently than agents without attitude and adapt more easily to dynamic situations. Another unique idea presented in this paper is that to deal quantitatively the imprecision or uncertainty of multiple attributes of items to acquire in auctions, fuzzy set technique is used. The fuzzy logic provides attitude based agents provide resources in the decision making process of bidding agent. The bidding strategy also allows for flexible heuristics both for the overall gain and for individual attribute evaluations. It also explores the relationships between evaluations of different attributes using Analytic Hierarchy Process method [Saaty, 1980].

There are a number of areas of further investigation. In future we would further like to explore the development of strategies for multiple auctions. We would also like to compare our bidding techniques with other decision theoretic approaches to determine the relative strengths and weaknesses of these methods. Different strategies may perform well in some environments but may perform poorly in another. The numbers of strategies that can be employed are endless and the search space is huge. To address this issue, we intend to use learning techniques to obtain a model of the price dynamics based on the past data and to search for most successful strategies in predefined environments in an offline fashion.

\section{REFERENCES}

Anthony, P. and N.R.Jennings (2002). Evolving bidding strategies for multiple auctions. In Proceedings of 15th European Conference on Artificial Intelligence, pages 187-192, Netherlands.

Byde, A., Priest, C., and N.R.Jennings (2002). Decision procedures for multiple auctions. pages 613-620.

Delgado, M., Herrera, F., and Herrera-Viedma, E. (2001). Combining linguistic information in a distributed intelligent agent model for information gathering on the internet. In Wang, P., editor, Computing with words, pages 251-276. John Wiley and Sons, Inc.

Delgado, M., Herrera, F., Herrera-Viedma, E., Verdegay, J. L., and Vila, M. A. (1999). Aggregation of linguistic information based on a symbolic approach. In Zadeh, L. and Kacpryzk,
J., editors, Computing with Words in Information/Intelligent Systems I. Foundations, pages 428-440. Physica-Verlag.

Greenwald, A. and Stone, P. (2001). Autonomous bidding agents in the trading agent competition. IEEE Internet Computing, pages 52-60.

He, M., Leung, H., and Jennings, N. (2003). A fuzzylogic based bidding strategy for autonomous agents in continuous double auctions. IEEE Transactions on Knowledge and data Engineering, 15(6):1345-1363.

Herrera, F., Herrera-Viedma, E., and Chiclana, F. (2001). Multiperson decision-making based on multiplicative preference relations. European J. Operational Research, 129:372-385.

Kowalcyzk, R. and Bui, V. (2000). On fuzzy e-negotiation agents: Autonomous negotiation with incomplete and imprecise information. In Proceedings Dexa e-Negotiation Workshop.

Luo, X., Jennings, N., Shadbolt, N., Leung, H., and Lee, J. (2003). A fuzzy constraint based model for bilateral, multi-issue negotiation in semi-competitive environments. Artificial Intelligence, 148(1-2):53-102.

Ma, H. and Leung, H.-F. (2007). An adaptive attitude bidding strategy for agents in continuous double auctions. Electronic Commerce Research and Applications, 6:383-398.

P.Anthony and N.R.Jennings (2003). Developing a bidding agent for multiple heterogeneous auctions. ACM transactions on Internet Technology, 3(3):185-217.

P.Stone, Littman, M., S.Singh, and M.Kearns (2001). Attac-2000: An adaptive autonomous bidding agent. Journal of Artificial Intelligence Research, 15:189-206.

Saaty, T. (1980). The Analytic Hierarchy Process. McGraw Hill, NY.

Yager, R. R. (1993). Families of OWA operators. Fuzzy Sets and Systems, 59:125-148.

Yager, R. R. (2004). OWA aggregation over a continuous interval argument with applications to decision making. IEEE Transactions on Systems, Man, and Cybernetics-Part B: Cybernetics, 34(5):1952-1963. 